\title{
The D-term form factor from dispersion relations in deeply virtual Compton scattering
}

\section{B. Pasquini*}

Dipartimento di Fisica, Università degli Studi di Pavia, INFN, Sezione di Pavia - Pavia, Italy

E-mail: barbara.pasquini@unipv.it

\begin{abstract}
We review a dispersive calculation of the D-term form factor, using unsubtracted $t$-channel dispersion relations for the deeply virtual Compton scattering amplitudes. The $t$-channel unitarity relation is saturated with the contribution of two-pion intermediate states, using the two-pion generalized distributions amplitude for the $\gamma^{*} \gamma \rightarrow \pi \pi$ subprocess and reconstructing the $\pi \pi \rightarrow N \bar{N}$ subprocess from available information on pion-nucleon partialwave helicity amplitudes. The dispersion-relation results are discussed in comparison with available model predictions and phenomenological extractions.
\end{abstract}

23rd International Spin Physics Symposium - SPIN2018 -

10-14 September, 2018

Ferrara, Italy

${ }^{*}$ Speaker. 


\section{Introduction}

Dispersion Relations (DRs) have seen various applications to study the electromagnetic structure of hadrons. In particular, DRs for Compton scattering processes, with both real and virtual photons, have been proven to be an useful tool for the prediction and extraction from experimental data of low-energy properties of hadron systems [1-6]. More recently, the dispersion formalism has been extended to describe the virtual Compton scattering process in the deep inelastic region [7-11]. In particular, it was shown that the amplitudes for Deeply Virtual Compton Scattering (DVCS) satisfy subtracted DRs at fixed $t$ with the subtraction function defined by the $D$-term Form Factor (FF) $[9,10]$.

The $D$-term FF was originally introduced to complete the parametrization of the Generalized Parton Distributions (GPDs) in hard exclusive reactions in terms of double distributions, and restore the polynomiality property of the singlet moments of unpolarized GPDs [12]. On the other side, the $D$-term FF enters the parametrization of the non-forward matrix element of the nucleon Energy-Momentum Tensor (EMT) and, as such, provides the key to introduce mechanical properties of the nucleon $[13,14]$.

Here we review the dispersive representation of the D-term FF in terms of DRs in the $t$-channel, as originally proposed in Ref. [15], providing a microscopic interpretation of the physical content of the D-term FF in terms of $t$-channel exchanges with the appropriate quantum numbers.

\section{2. $t$-channel dispersion relations for the D-term FF}

We consider the DVCS process $\gamma^{*}(q) N(p) \rightarrow \gamma\left(q^{\prime}\right) N\left(p^{\prime}\right)$. The familiar Mandelstam variables are $s=(p+q)^{2}, t=\left(q-q^{\prime}\right)^{2}, u=\left(q-p^{\prime}\right)^{2}$, and are constrained by $s+u+t=$ $2 M_{N}^{2}-Q^{2}$, with $M_{N}$ the nucleon mass and $Q^{2}=-q^{2}$.

We consider the Bjorken regime, where the photon virtuality $Q^{2}$ and $s$ are large, and $-t \ll s, Q^{2}$. We discuss here the use of DRs for the DVCS amplitude related to the GPD $E$ for unpolarized quarks in a transversely polarized nucleon target. This amplitude, denoted as $F$, is function of the crossing variable $\nu=(s-u) / 4 M_{N}, t$, and $Q^{2}$. In the $\nu-t$ plane at fixed $Q^{2}, F$ satisfies the following fixed- $t$ subtracted DR [9-11]:

$$
F^{q}(\nu, t)=F^{q}(0, t)+\frac{\nu^{2}}{\pi} \int_{\nu_{0}}^{\infty} \frac{\mathrm{d} \nu^{\prime 2}}{\nu^{\prime 2}} \frac{\operatorname{Im} F^{q}\left(\nu^{\prime}, t\right)}{\nu^{\prime 2}-\nu^{2}},
$$

where $\nu_{0}=Q^{2} / 4 M_{N}$. The subtraction function $F^{q}(0, t)$ can be related to the D-term as follows

$$
F^{q}(0, t)=2 \int_{-1}^{+1} \mathrm{~d} z \frac{\mathcal{D}^{q}(z, t)}{1-z}=4 \mathcal{D}(t) .
$$

Using the expansion of the D-term $\mathcal{D}(z, t)$ in terms of Gegenbauer polynomials, one obtains the following series for the D-term FF:

$$
\mathcal{D}^{q}(t)=\sum_{\substack{n=1 \\ n \text { odd }}}^{\infty} d_{n}^{q}(t) .
$$


The dispersive representation for the D-term FF $\mathcal{D}(t)$ of Eq. (2.2) can be obtained by applying unsubtracted DRs in the variable $t$ :

$$
F^{q}(0, t)=\frac{1}{\pi} \int_{4 m_{\pi}^{2}}^{+\infty} \mathrm{d} t^{\prime} \frac{\operatorname{Im}_{t} F^{q}\left(0, t^{\prime}\right)}{t^{\prime}-t}+\frac{1}{\pi} \int_{-\infty}^{-a} \mathrm{~d} t^{\prime} \frac{\operatorname{Im}_{t} F^{q}\left(0, t^{\prime}\right)}{t^{\prime}-t}
$$

The second integral in Eq. (2.4) extends from $-\infty$ to $-a=-2\left(m_{\pi}^{2}+2 M_{N} m_{\pi}\right)-Q^{2}$. As we are interested in evaluating Eq. (2.4) for large $Q^{2}$ values and small (negative) values of $t$ $(|t| \ll a)$, the integral from $-\infty \rightarrow-a$ is suppressed, and will be neglected. The imaginary part in the integral from $4 m_{\pi}^{2} \rightarrow+\infty$ in Eq. (2.4) is saturated by the possible intermediate states for the $t$-channel process, which lead to cuts along the positive- $t$ axis. For low values of $t$, the $t$-channel discontinuity is dominated by $\pi \pi$ intermediate states. Consequently, we shall saturate the integral in Eq. (2.4) by the contribution of the $\gamma^{*} \gamma \rightarrow \pi \pi \rightarrow N \bar{N}$ channel, which turns out to be a good approximation for small $t$. The $\gamma^{*} \gamma \rightarrow \pi \pi$ subprocess at large $Q^{2}$ and small $t$ can be described in a factorized form as the convolution of a short-distance contribution, $\gamma^{*} \gamma \rightarrow q \bar{q}$, perturbatively calculable, and nonperturbative matrix elements which describe the exclusive fragmentation of a $q \bar{q}$ pair into two pions and are given in terms of two-pion Generalized Distribution Amplitudes (GDAs). We use a partial wave expansion of the GDAs, restricting ourselves to the contribution of the $S$ - and $D$-wave amplitudes. This corresponds to consider the term with $n=1$ in Eq. (2.3), i.e. to evaluate only the contribution from the $\mathrm{FF} d_{1}(t)^{q}$ in Eq. (2.2). Furthermore, the two-pion GDAs are calculated through dispersion relations using the Omnès representation which was first discussed in Ref. [13]. This information is then combined with the $\pi \pi \rightarrow N \bar{N}$ partial wave amplitudes, which are determined by analytical continuation of the $\pi N$ scattering amplitudes [16]. As final result, we obtain

$$
\operatorname{Im}_{t} F^{q(\pi \pi)}=\frac{3 M_{N} p_{\pi}}{2 \sqrt{t} p_{t}^{2}} B_{12}^{q}(0)\left[\left(3 C-\beta^{2}\right) f_{0}(t) f_{+}^{0 *}(t)+\left(p_{\pi} p_{t}\right)^{2} \beta^{2} f_{2}(t) f_{+}^{2 *}(t)\right]
$$

where $p_{t}=\sqrt{t / 4-M_{N}^{2}}$ and $p_{\pi}=\sqrt{t / 4-m_{\pi}^{2}}$. In Eq. (2.5), the Omnès functions $f_{0,2}$ and the $\pi N$ amplitudes $f_{+}^{0,2}$ are calculated in terms of the same $\pi \pi$ phase shifts. Furthermore, the constants $C$ and $B_{12}$ come from the Gegenbauer expansion of the two-pion GDAs. $C$ is taken from the instanton model at low energies [17], giving $C=1+b m_{\pi}^{2}+\mathcal{O}\left(m_{\pi}^{4}\right)$ with $b \approx-1.7 \mathrm{GeV}^{-2}$, and the coefficient $B_{12}(0)$ is obtained using the crossing relations between the quark $2 \pi \mathrm{DA}$ 's and the corresponding parton distributions in the pion, i.e.

$$
B_{12}^{q}(0)=\frac{10}{9} \int \mathrm{d} x x \frac{1}{N_{f}} \sum_{f}\left[q_{\pi}^{f}(x)+\bar{q}_{\pi}^{f}(x)\right] .
$$

We note that in Eq. (2.5), the dependence on the renormalization scale enters only through the coefficient $B_{12}^{q}$ evaluated at $t=0$, and therefore is disjoined from the $t$ dependence of the amplitude. Furthermore, the coefficients $B_{12}^{q}$ evolve in the same way as the quark momentum fraction in the pion, in accordance with Eq. (2.6). 


\section{Results}

The $d_{1}(t) \mathrm{FF}$ is particularly interesting, as it enters the parametrization of the EMT of QCD. In particular, the relation to the EMT FF reads as $D(t)=\frac{4}{5} d_{1}(t)$. The physical content encoded in the EMT FFs is revealed in the so-called Breit frame $[13,14]$ and has been discussed recently in other frames in Refs. [18,19]. Working in the Breit frame, the $D(t)$ can be related to the spatial distribution of shear forces $s(r)$ and pressure $p(r)$ as

$p(r)=\frac{1}{3} \int \frac{\mathrm{d}^{3} \vec{\Delta}}{2 m(2 \pi)^{3}} e^{-i \vec{\Delta} \cdot \vec{r}} P_{0}(\cos \theta)[t D(t)], \quad s(r)=\frac{3}{4} \int \frac{\mathrm{d}^{3} \vec{\Delta}}{2 m(2 \pi)^{3}} e^{-i \vec{\Delta} \cdot \vec{r}} P_{2}(\cos \theta)[t D(t)]$,

where $t=\vec{\Delta}^{2}$. The relation for the shear forces holds for quark and gluon separately, while it is defined only for the total system in the case of the pressure. Thanks to Eq. (3.1), the EMT FF introduces to the mechanical properties of the nucleon and reflects the internal dynamics of the system through the distribution of forces. Requiring that for the mechanical stability of the system the corresponding force must be directed outwards, one expects the local criterion $2 s(r)+p(r)>0$, which implies that the $D$-term for any stable system must be negative, $D<0$, as confirmed in models [20-22], calculations from DRs [15] and lattice QCD [23] for the nucleon. Another consequence of the EMT conservation is the von Laue condition, which shows how the internal forces balance inside a composed particle, i.e.,

$$
\int_{0}^{\infty} p(r) r^{2} \mathrm{~d} r=0
$$

This relation implies that the pressure must have at least one node. In all model studies so far it was found that the pressure is positive in the inner region, and negative in the outer region, with the positive sign meaning repulsion towards outside and the negative sign meaning attraction directed towards inside.

Recently, an analysis of the published JLab data measured at $6 \mathrm{GeV}[26,27]$ has provided experimental information on the total quark contribution to the $D(t) \mathrm{FF}$ of the nucleon [24], as shown in Fig. 1 (squared in the left panel, referring to a scale of $\mu^{2}=1.5 \mathrm{GeV}^{2}$ ), in comparison with the KM15 fit [25] and calculations from DRs [15] and lattice QCD [23] (all to the scale of $4 \mathrm{GeV}^{2}$ ) and scale independent results from the bag [20], chiral quark soliton [21] and Skyrme [22] models. The $D$-term parameters fitted to the JLab data, with the assumption of a negligible gluon contribution, were used to plot the radial pressure distribution shown by the black solid curve in the right panel of Fig. 1. The corresponding estimated uncertainties are displayed as the light-green shaded area. The blue area represents the uncertainties from all the data that were available before the $6-\mathrm{GeV}$ experiments, and the red shaded area shows projected results from future JLab experiments at $12 \mathrm{GeV}$. Within the uncertainties of the analysis, the distribution satisfies the stability condition (3.2), with a zero crossing near $r=0.6 \mathrm{fm}$.

The first extraction of the pion EMT form factor from the BELLE data on $\gamma^{*} \gamma \rightarrow \pi^{0} \pi^{0}$ was reported in Ref. [28]. The result at zero momentum transfer is $D^{Q}(0) \approx-0.75$, in agree- 

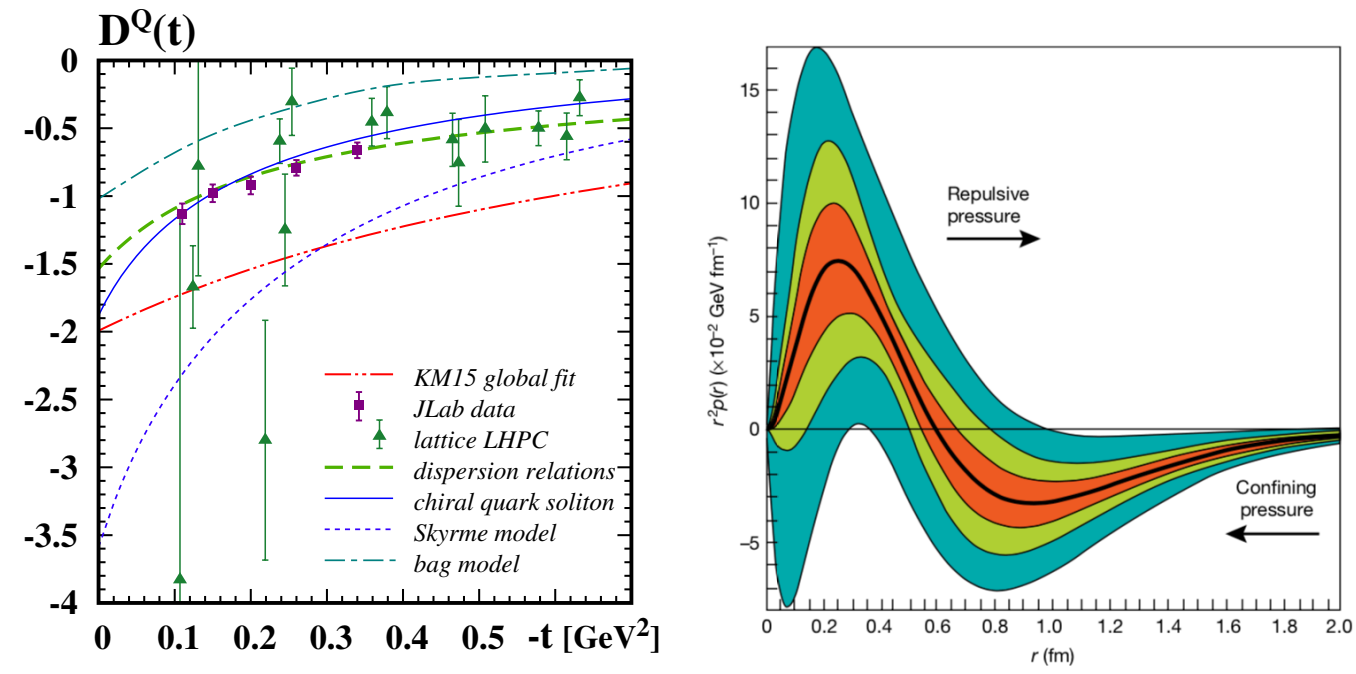

Figure 1: Left (from Ref. [14]): The $D^{Q}(t) \mathrm{FF}$ from the JLab analysis [24], in comparison with the KM15 fit [25] and calculations from DRs [15] and lattice QCD [23], and results from the bag [20], chiral quark soliton [21] and Skyrme [22] model. Right (from Ref. [24]): quark contribution to the pressure distribution $r^{2} p(r)$ as function of the radial distance $r$ from the centre of the proton.

ment with the soft pion theorem $D=-1$, given that the extracted value does not include the gluon contribution. The extraction also shows that $D$-term is definitely negative, as expected for the mechanical stability of the pion.

\section{References}

[1] B. Pasquini and M. Vanderhaeghen, Dispersion Theory in Electromagnetic Interactions, Ann. Rev. Nucl. Part. Sci. 68 (2018) 75.

[2] D. Drechsel, B. Pasquini and M. Vanderhaeghen, Dispersion relations in real and virtual Compton scattering, Phys. Rept. 378 (2003) 99.

[3] B. Pasquini, M. Gorchtein, D. Drechsel, A. Metz and M. Vanderhaeghen, Dispersion relation formalism for virtual Compton scattering off the proton, Eur. Phys. J. A11 (2001) 185.

[4] D. Drechsel, M. Gorchtein, B. Pasquini and M. Vanderhaeghen, Fixed $t$ subtracted dispersion relations for Compton Scattering off the nucleon, Phys. Rev. C 61 (1999) 015204.

[5] B. Pasquini, D. Drechsel and M. Vanderhaeghen, Proton spin polarizabilities from polarized Compton scattering, Phys. Rev. C 76 (2007) 015203.

[6] B. Pasquini, D. Drechsel and S. Scherer, The Polarizability of the pion: No conflict between dispersion theory and chiral perturbation theory, Phys. Rev. C77 (2008) 065211.

[7] D. Müller and K. M. Semenov-Tian-Shansky, $J=0$ fixed pole and D-term form factor in deeply virtual Compton scattering, Phys. Rev. D92 (2015) 074025.

[8] K. Kumericki, D. Mueller and K. Passek-Kumericki, Towards a fitting procedure for deeply virtual Compton scattering at next-to-leading order and beyond, Nucl. Phys. B794 (2008) 244. 
[9] I. V. Anikin and O. V. Teryaev, Dispersion relations and subtractions in hard exclusive processes, Phys. Rev. D76 (2007) 056007.

[10] A. V. Radyushkin, Generalized Parton Distributions and Their Singularities, Phys. Rev. D83 (2011) 076006.

[11] M. Diehl and D. Yu. Ivanov, Dispersion representations for hard exclusive processes: beyond the Born approximation, Eur. Phys. J. C52 (2007) 919.

[12] M. V. Polyakov and C. Weiss, Skewed and double distributions in pion and nucleon, Phys. Rev. D60 (1999) 114017.

[13] M. V. Polyakov, Generalized parton distributions and strong forces inside nucleons and nuclei, Phys. Lett. B555 (2003) 57.

[14] M. V. Polyakov and P. Schweitzer, Forces inside hadrons: pressure, surface tension, mechanical radius, and all that, Int. J. Mod. Phys. A33 (2018) 1830025.

[15] B. Pasquini, M. V. Polyakov and M. Vanderhaeghen, Dispersive evaluation of the D-term form factor in deeply virtual Compton scattering, Phys. Lett. B739 (2014) 133.

[16] G. Höhler, Pion-Nucleon Scattering, vol. I/9b2. Landolt-Börnstein, H. Schopper, Springer, 1983.

[17] M. V. Polyakov and C. Weiss, Two pion light cone distribution amplitudes from the instanton vacuum, Phys. Rev. D59 (1999) 091502.

[18] C. Lorcé, H. Moutarde and A. P. Trawiński, Revisiting the mechanical properties of the nucleon, Eur. Phys. J. C79 (2019) 89.

[19] C. Lorcé, L. Mantovani and B. Pasquini, Spatial distribution of angular momentum inside the nucleon, Phys. Lett. B776 (2018) 38.

[20] X.-D. Ji, W. Melnitchouk and X. Song, A Study of off forward parton distributions, Phys. Rev. D56 (1997) 5511.

[21] K. Goeke, J. Grabis, J. Ossmann, M. V. Polyakov, P. Schweitzer, A. Silva et al., Nucleon form-factors of the energy momentum tensor in the chiral quark-soliton model, Phys. Rev. D75 (2007) 094021.

[22] C. Cebulla, K. Goeke, J. Ossmann and P. Schweitzer, The Nucleon form-factors of the energy momentum tensor in the Skyrme model, Nucl. Phys. A794 (2007) 87.

[23] LHPC collaboration, P. Hagler et al., Nucleon Generalized Parton Distributions from Full Lattice QCD, Phys. Rev. D77 (2008) 094502.

[24] V. D. Burkert, L. Elouadrhiri and F. X. Girod, The pressure distribution inside the proton, Nature 557 (2018) 396.

[25] K. Kumericki and D. Müller, Description and interpretation of DVCS measurements, EPJ Web Conf. 112 (2016) 01012.

[26] CLAS collaboration, F. X. Girod et al., Measurement of Deeply virtual Compton scattering beam-spin asymmetries, Phys. Rev. Lett. 100 (2008) 162002.

[27] CLAS collaboration, H. S. Jo et al., Cross sections for the exclusive photon electroproduction on the proton and Generalized Parton Distributions, Phys. Rev. Lett. 115 (2015) 212003.

[28] S. Kumano, Q.-T. Song and O. V. Teryaev, Hadron tomography by generalized distribution amplitudes in pion-pair production process $\gamma^{*} \gamma \rightarrow \pi^{0} \pi^{0}$ and gravitational form factors for pion, Phys. Rev. D97 (2018) 014020. 